\title{
The time course of spatial memory distortions
}

\author{
STEFFEN WERNER \\ University of Idaho, Moscow, Idaho \\ and \\ JÖRN DIEDRICHSEN \\ University of California, Berkeley, California
}

\begin{abstract}
Four experiments investigated the memory distortions for the location of a dot in relation to two horizontally aligned landmarks. In Experiment 1, participants reproduced from memory a dot location with respect to the two landmarks. Their performance showed a systematic pattern of distortion that was consistent across individual participants. The three subsequent experiments investigated the time course of spatial memory distortions. Using a visual discrimination task, we were able to map the emergence of spatial distortions within the first $800 \mathrm{msec}$ of the retention interval. After retention intervals as brief as $50 \mathrm{msec}$, a distortion was already present. In all but one experiment, the distortion increased with longer retention intervals. This early onset of spatial memory distortions might reflect the almost immediate decay of detailed spatial information and the early influence of an enduring spatial memory representation, which encodes spatial information in terms of the perceived structure of space.
\end{abstract}

Remembering the location of an object in space is one of the elementary problems in spatial cognition and critical to a large number of spatially oriented actions. Interestingly, a large body of psychological research exists showing that people often err systematically when remembering spatial locations. These distortions, or biases, in spatial memory are commonly seen as evidence that the perceived structure of space (e.g., different regions, groupings, etc.) biases the way in which locations are remembered. A simple experiment by Huttenlocher, Hedges, and Duncan (1991; see also Laeng, Peters, \& McCabe, 1998) illustrates this point. When observers see a dot within a circle and later have to reproduce the location of the dot within a second circle, they show a systematic pattern of errors. Dots close to the imagined vertical or horizontal meridian of the circle are reproduced at angles away from the two axes, exaggerating small deviations. Spatial distortions such as these appear to be ubiquitous and can be found whenever space is structured in some way. Even children as young as 16 months exhibit spatial behavior that indicates distortions in spatial memory. When toddlers are searching for a buried toy, for example, they start digging at locations systematically drawn toward the center of a rectangular sandbox (Huttenlocher, Newcombe, \& Sandberg, 1994). Other research has demonstrated spatial memory distortions in a variety of domains, ranging from the orientation of real or virtual

This research was supported by a research grant of the German Science Foundation to S.W. We thank Björn Rump for his assistance in gathering and analyzing the data. We are also thankful to Thomas Schmidt, Michael Waldmann, and William Prinzmetal for their helpful comments on earlier drafts of this article. Correspondence should be addressed to S. Werner, Department of Psychology, University of Idaho, Moscow, ID 83844-3043 (e-mail: swerner@uidaho.edu). lines (Bryant \& Subbiah, 1993; Schiano \& Tversky, 1992) to large-scale or geographical relations (Hirtle \& Jonides, 1985; Stevens \& Coupe, 1978; Tversky, 1981).

As results by Crawford, Huttenlocher, and Engebretson (2000) indicate, the spatial distortions discussed above are not simply due to perceptual biases. In their study, observers had to adjust the length of a reference line to the perceived length of a Müller-Lyer stimulus. Participants either adjusted the line while the Müller-Lyer stimulus was in view or reproduced the length from memory. In both cases, similar biases consistent with the Müller-Lyer effect were found, confirming the role of early stages of perceptual processing in this illusion (Moore \& Egeth, 1997). However, only in the memory condition was there an additional distortion of reproduced line length toward the average line length presented in the course of the experiment. Crawford et al. consider this context set effect as an example of the distortions introduced by reconstructive processes during memory retrieval.

The model of spatial memory distortionsby Huttenlocher et al. (1991) offers an explanation for such reconstructive distortion effects in spatial memory. The model assumes that spatial memory distortions arise from the combination of multiple sources of information. They propose that memory for spatial locations, for example, is encoded in two separate ways. A fine-grained coordinate representation of location encodes the location independently of the perceived structure of the space. A second, categorical, representation stores the spatial category (e.g., a region) to which the location belongs together with a prototypical location for this category (e.g., a central point of that region). Only this second representation is sensitive to the way space is structured by the observer. Distortions arise in the model whenever fine-grained coordinate information is uncer- 
tain and a location has to be inferred from its spatial category membership. According to this reconstructive account, the remembered location will be partially guessed on the basis of the prototypical location of the remembered category to which the location belongs. Remembered locations are thus repelled from a border toward the category prototype, the size of the distortion depending on the relative weight of the source of information.

Very little is known to date about the exact mechanism that gives rise to these ubiquitous distortions. One important piece of information, which may constrain theoretical models of spatial memory distortions, concerns the question of the temporal characteristics of these distortions. The studies previously mentioned provide only a rough idea about the precise point in time at which spatial memory distortions are already present. In experiments using the dot location and similar tasks, a few seconds, consisting of retention interval and reproduction time, were sufficient to produce significant spatial distortions (Laeng et al., 1998; Nelson \& Chaiklin, 1980). In addition, Huttenlocher et al. (1991) demonstrated that a secondary task interfered with location memory, leading to larger distortions over the course of $10 \mathrm{sec}$. For very brief retention intervals, however, the studies previously described leave open two possible scenarios. First, spatial memory distortions may be present only after a significant retention interval (on the order of seconds), in accordance with the notion that location information enters short-term memory as it is perceived and is subsequently subjected to memory distortions. Second, spatial distortions may also develop very rapidly within the first few hundred milliseconds, indicating that accurate location information is lost very quickly. In either case, the Huttenlocheret al. model would explain the distortion effect by a loss of detailed coordinate information and a heavier reliance on categorical information. Information about the life span of the hypothesized fine-grained coordinate information would thus serve to characterize this representation.

Historically, the temporal change of contents in visual memory has played an important role in theories of form perception and memory proposed by the Gestalt psychologists (see Riley, 1962). Early studies indicated that visual memory for form as measured through successive reproductions showed evidence of systematic distortions (Wulff, 1922). The nature and the underlying principles of these distortions remained largely unclear (see Carmichael, Hogan, \& Walter, 1932, for the role of the semantic interpretation). The time course of these types of distortions of memory for visual form was often investigated for long retention intervals (up to many weeks). Interestingly, Crumbaugh (1954) investigated the time course of distortions of visual form memory within the first $12 \mathrm{sec}$ after presentation of the original stimulus using a visual discrimination task. For some of his stimuli, marked distortions were noticeable within the first second after presentation and increased only slightly with growing retention interval. Unfortunately, his results do not paint a consistent picture, because some of the changes in distortions seemed to asymptote after $1 \mathrm{sec}$ whereas others appeared to develop much later.

That accurate information can be lost even within short retention intervals was supported in a more recent study on the time course of representational momentum (Freyd \& Johnson, 1987). In this experiment, participants saw a succession of rectangles, which induced the perception of rotational motion of the rectangle. After the presentation of the last rectangle of the sequence, one of nine different rectangles was displayed and the participants had to indicate whether the orientation of the probe corresponded to the last rectangle they had seen. The nine different probes varied in their orientation between $-8^{\circ}$ and $8^{\circ}$ in steps of $2^{\circ}$ from the correct orientation. By comparing the rates of same responses for each probe orientation, the authors were able to estimate the amount of distortion for different retention intervals. These results suggest that spatial distortions in the direction of the perceived movement developed within the first $400 \mathrm{msec}$ after offset of the visual stimulus. More generally this result shows that spatial distortions might arise at brief exposures and retention intervals of spatial information. Obviously, the traditional task of reproducing a remembered target location is not sufficiently fast within these temporal parameters of only a few hundred milliseconds for investigating the development of spatial distortions. The method employed by Freyd and Johnson and earlier by Crumbaugh (1954), on the other hand, provides a promising paradigm for tapping into the temporal dynamics of the development of spatial distortions. In this article we will address the development of spatial distortions in the course of spatial information processing by using a similar discrimination paradigm.

The investigation of the time course of spatial memory distortions requires that the distortion itself be reliable. In Experiment 1 we therefore first report a pattern of distortions that is reliable across observers for a simple spatial configuration, namely the location of a dot in relation to two horizontally aligned landmarks. Participants had to reproduce the location of the dot in relation to the two landmarks after a 2 -sec retention interval on the computer screen. In the subsequent three experiments we probed the temporal characteristics of different aspects of this empirical distortion pattern.

\section{EXPERIMENT 1}

Very different mechanisms and systems of reference can influence how space is structured. Systems of reference can be defined in relation to the perceiver or to different systems of landmarks (see Levinson, 1996, for an overview). In our experiment, we used two horizontally aligned landmarks to control the perceptual organization of visual space. A two-landmark display is the simplest geometric configuration that defines a reference frame with location, orientation, and scale. We chose this configuration over a circle (e.g., Huttenlocher et al., 1991) because it allows the manipulation of the internal orientation of the stimulus independent of the observer (Werner \& Schmidt, 2000). 
The task of the participants was to reproduce a dot location with respect to the two landmarks. Two additional reference systems could be used in this situation. First, participants could try to encode the location of the target dot with respect to the visible frame of the monitor, or, alternatively, participants could try to encode the dot location in an egocentric frame of reference. Because we are interested exclusively in the pattern of distortions evoked by the coding relative to the two horizontally aligned landmarks, we included two conditions in the experiment. In one condition, the landmarks were always presented at the same location, allowing the participants to represent the location relative to the frame of the monitor or in egocentric coordinates. In a second condition, we varied the position of the landmarks between presentation and reproduction, thus leaving the two landmarks as the only reliable reference system. In both conditions, the participants were instructed to reproduce the target relative to the landmarks. Any differences between these conditions could be attributed to the use of different reference systems.

\section{Method}

Participants. Twelve students at the University of Göttingen (7 female, 5 male) participated in this study in return for $30 \mathrm{DM}$ payment. Their age ranged from 19 to 35 years.

Apparatus and Stimuli. The stimuli were presented on a 14-in. Apple RGB-monitor $(640 \times 480$ pixel resolution, 100 pixel $\approx 3.67 \mathrm{~cm})$ viewed from a distance of $90 \mathrm{~cm}$. The background color of the mon- itor was set to black and all stimuli were presented in white. Two circles served as landmarks, each 10 pixels $\left(0.23^{\circ}\right)$ in diameter and presented 200 pixels $\left(4.67^{\circ}\right)$ apart from each other horizontally. The target dot was a small filled white circle with a diameter of 5 pixels $\left(0.11^{\circ}\right)$ at 1 of 125 locations. The locations were symmetrically organized, covering an area including the two landmarks on a regular grid with 25 columns and 5 rows, spanning an area of $240 \times 60$ pixels $\left(5.60^{\circ} \times\right.$ $1.40^{\circ}$; see Figure 1 for details). To reproduce a dot location, a cursor of the same size and shape as the dot could be moved across the screen.

Procedure. Participants were randomly assigned to one of two conditions. In the fixed-landmarks condition, the two landmarks were always presented centered on the screen. In the variable-landmarks condition, for both presentation and reproduction, the landmarks were shifted horizontally and vertically by a random value between -150 and 150 pixels $\left(3.7^{\circ}\right)$ relative to the fixed-landmark condition.

The experiment consisted of two 1-h sessions, separated by at least 1 day. In each session, a participant was presented 250 trials, so that each location was tested a total of four times per participant. Every trial began with the presentation of the landmarks and the dot for $1.5 \mathrm{sec}$, followed by a retention interval of $2 \mathrm{sec}$, in which the screen was left blank. The simultaneous reappearance of the landmarks and the mouse pointer signaled the participants to move the mouse pointer to the remembered position of the dot relative to the landmarks. They finished each trial by pressing the mouse button. After an interval of $1 \mathrm{sec}$ the next trial started.

\section{Results}

All trials in which the reproduced position deviated more that 20 pixels $\left(0.47^{\circ}\right)$ from the correct location were considered outliers and were excluded from further analysis
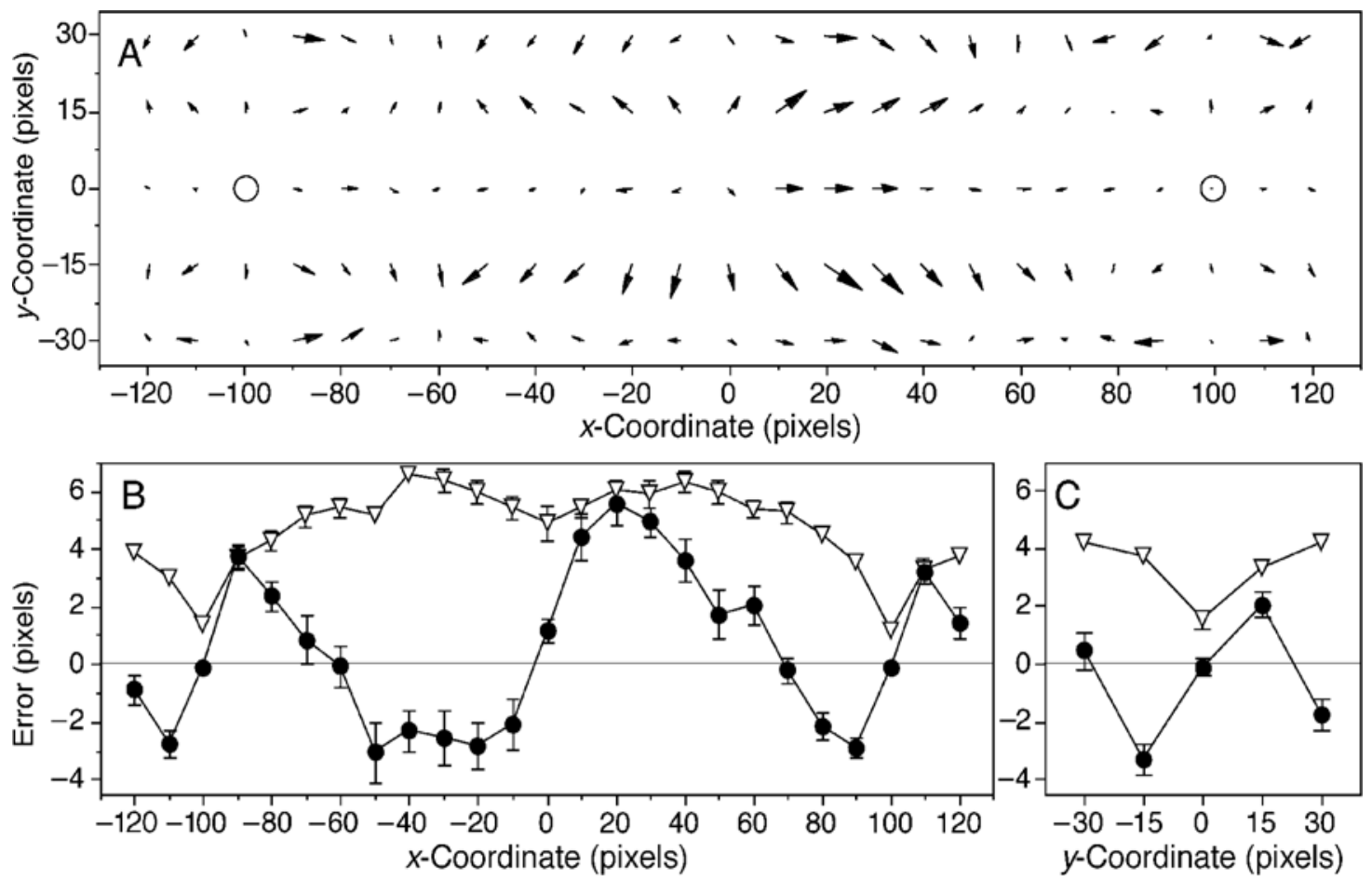

Figure 1. (A) Vector plot of the distortions in reproduction in Experiment 1 with two horizontally aligned landmarks (circles). The arrows start at the presented location and end at the averaged reproduced location. (B) Mean constant (filled circles) and variable error (open triangles) in the horizontal direction $( \pm S E)$ dependent on the horizontal location of the point. Positive constant error indicates a rightward distortion. (C) Mean constant and variable error in the vertical direction. Positive constant error indicates an upward distortion. 
(2.33\%). For each location and each participant, the constant error was calculated as the mean difference between presented and reproduced location. The variable error was defined as the standard deviation of these differences across the different trials of each participant. Average constant errors at the 125 locations are plotted in Figure 1A.

Figure 1B shows the constant and variable error for the horizontal direction collapsed over the vertical direction. The horizontal constant error was submitted to a mixed analysis of variance (ANOVA) with $x$-coordinate as a within-subjects and the landmark condition (fixed vs. variable landmarks) as a between-subjects variable. The constant error varied systematically with the $x$-coordinate $\left[F(24,240)=17.10, M S_{\mathrm{e}}=5.17, p<.001\right]$. Neither the effect of landmark condition nor its interaction with the $x$ coordinate, was significant (both $F \mathrm{~s}<1$ ). A similar picture was obtained in the corresponding analysis for the vertical axis (Figure 1C). The $y$-coordinate had a significant effect on the constant error in the vertical direction $\left[F(4,40)=15.10, M S_{\mathrm{e}}=3.38, p<.001\right]$, and again the landmark condition did not have an effect on the constant error, nor was there a significant interaction between these variables.

The variable error on both dimensions was dependenton the position of the dot on that dimension $[F(24,240)=$ $25.52, M S_{\mathrm{e}}=1.03, p<.001$, for the horizontal dimension and $F(4,40)=77.39, M S_{\mathrm{e}}=0.186, p<.001$, for the vertical dimension]. The variable error in the horizontal dimension was reduced near the landmarks and near the midpoint between the landmarks, as well as in the vertical dimension on the imaginary line connecting the two landmarks. There was no difference between the variable- and fixed-landmarks groups in terms of variable error (both $F_{\mathrm{S}}<1$ ), nor was there any significant interaction with the position of the dot. Thus, our data show that moving the landmarks between presentation and reproduction did not have any effect.

\section{Discussion}

When reproducing the location of a dot in a simple configuration of two horizontally aligned landmarks, participants showed a stable, symmetric pattern of distortions. Dots presented near a landmark were remembered further away from the landmark, whereas dots presented near the midpoint between the two landmarks were reproduced further away from the midpoint. This observation also holds for the vertical direction. The individual distortion patterns were stable across participants. The average correlation between each individual pattern of constant errors with the averaged pattern of the remaining participants was $r=.79$ for the horizontal and $r=.89$ for the vertical direction (averaged across participants).

The failure to find any differences between the variablelandmarks conditions and the fixed-landmarks condition suggests that the participants encoded the target dot location relative to the landmarks. However, this finding alone does not rule out the use of an egocentric reference frame by the observer. An observer could, for example, use the distance and direction from the point of fixation on a landmark as an egocentric reference system to encode the position of the target location. Results from another series of experiments in which only one of the two landmarks was presented unpredictably during reproduction render this hypothesis unlikely (Schmidt, Werner, \& Diedrichsen, 2002). In addition, other studies suggest that the general pattern of distortions is robust with respect to rotating and scaling of the landmark configuration (Diedrichsen, 1999; Werner \& Schmidt, 2000), underlining the dependence of the distortion pattern on the landmarks.

Experiment 1 established an interindividually stable spatial distortion pattern induced by two horizontally aligned landmarks. Subsequent experiments focused on the temporal dynamics of the distortion pattern found in Experiment 1 to identify the retention interval at which accurate spatial information is lost by using a visual discrimination task. This information will help to constrain an important characteristic of proposed processes and representations for models of spatial memory distortions. In Experiment 2, location memory was tested for retention intervals of 100-800 msec for two different sets of target dots from Experiment 1, one that showed a large distortion and one that did not show any distortion. The results indicate that the distortion was present only for the former dots. In Experiment 3, the memory distortion for dots at these locations was detectable at retention intervals as short as $50 \mathrm{msec}$, and it depended on the presence of the landmark. We also assessed the effect of changing the expectations of the observers. The results suggest that manipulations of the response criterion do not impact the general pattern of our findings. Finally, in order to generalize our findings, in Experiment 4, we tested a set of target dots that showed distortions in the opposite direction in Experiment 1, using the same procedure as in Experiment 3.

\section{EXPERIMENT 2}

To get around the long response times of reproduction procedures, we employed a visual discrimination task in Experiments 2-4, presenting the dot twice in brief, masked succession. The task of our participants was to identify whether the dot changed its location between presentations. For their judgment, participants thus had to rely on their memory of the dot's location. Unlike Experiment 1, these experiments employed a masking procedure to eliminate apparent motion cues for brief retention intervals.

To infer the amount of the distortion from the participants' judgments, we devised a simple model of the processes involved in making a visual discrimination between two presented locations. The reproductions in Experiment 1 for the same location varied from trial to trial. This is partly due to noise introduced during the reproduction task, but also due to uncertainty about the exact remembered location. We therefore assume that a memory representation of the presented location corresponds to a distribution of locations with mean $\mu_{m}$ and variability $\sigma_{m}$. 


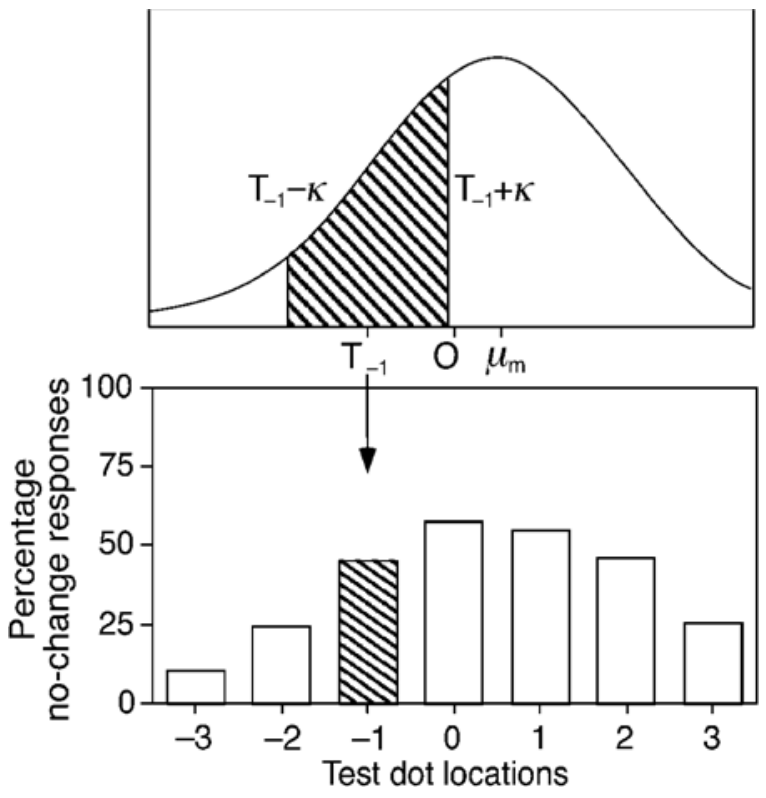

Figure 2. Model for the decision process in Experiments 2-4. The original dot is presented at location $O$ and the remembered location $m$ of this dot is normally distributed around $\mu_{m}$. In this example, the memory representation is distorted on average to the right. Presented with a test dot at location $T_{-1}$, the participant compares the test dot with the memory representation and gives a no-change response if the two are less different than a decision criterion $\kappa$ (i.e., if $m$ falls into the hatched area).

A memory distortion is present if $\mu_{m}$ is different from the original dot location. Consider now the example in Figure 2. Here the remembered location is biased on average to the right of the original location $O$. Presented with a test dot at the location $T_{-1}$, the participants will compare $T_{-1}$ with their remembered location and will give a no-change response if the two are sufficiently similar. We conceptualize this criterion in the decision process as a parameter $\kappa$. The hatched area in Figure 2 corresponds to the probability of the remembered location satisfying this criterion for the test dot $T_{-1}$ and therefore equals the probability of a no-change response in this case. By varying the position of the test dot, we can thus infer the average remembered location $\mu_{m}$, the accuracy of this location $\sigma_{m}$, and the decision criterion $\kappa$ from the rate of no-change responses (see Appendix for a detailed description).

In Experiment 2 we compared two kinds of dot positions: biased points, for which we had found a strong distortion in a certain direction on the basis of our data from Experiment 1 , and control points, for which we had not found any significant distortion. For both groups of points we varied the retention interval to map the time course of the memory distortion.

\section{Method}

Participants. Twelve students (7 male and 5 female) participated in two 1-h experimental sessions in return for $30 \mathrm{DM}$. Their age ranged between 19 and 28 years.
Apparatus and Stimuli. The experiment was carried out in a light-attenuated room. All stimuli were presented on a 16-in. Apple monitor $(832 \times 642$ pixel resolution) viewed from $90 \mathrm{~cm}$. All stimuli were made identical in size and shape to the ones used in Experiment 1 . We selected four dot positions that had shown a strong distortion in Experiment 1 (biased points $\{ \pm 20, \pm 15\}$; see Figure 3 ). As a control condition we also included three locations at which no distortion was found (control points $\{0, \pm 30\}$ and $\{0,0\}$ ). For each biased point we selected seven dot locations as test stimuli for the discrimination task. On the basis of the distortion of the original dot location found in Experiment 1, three of the locations were equally spaced in the direction of the distortion $(+1,+2,+3)$ and three in the opposite direction $(-1,-2,-3)$ in increments of 4.1 pixels $\left(.09^{\circ}\right)$. The distortion vectors were oriented in an approximately $45^{\circ}$ orientation and pointed away from the midpoint of the display. The center location (0) always was identical to the original location (Figure 3). Because the control points did not show a distortion in Experiment 1 , we used the same sets of test stimuli for the control points as for the biased points. Test dots oriented on a $45^{\circ}$ line from the upper left to the lower right were used for the upper $\{0,30\}$ and central control $\{0,0\}$ points, whereas test dots oriented from the upper left to the lower right were used for the lower $\{0,-30\}$ and central control points.

Procedure. Each trial began with a 200-msec presentation of the two landmarks and the dot and was followed by a masked retention interval of $100,200,400$, or $800 \mathrm{msec}$. In this interval, an area of $300 \times 200$ pixels $\left(7^{\circ} \times 4.6^{\circ}\right)$ was covered with 30 white lines of random position, orientation, and length (each 1 pixel wide), and neither the landmarks nor the dot were visible. The lines changed every $16 \mathrm{msec}$, covering $10 \%-15 \%$ of the area in every frame. Thereafter the two landmarks and the test dot were presented again for $200 \mathrm{msec}$. The participants were instructed to press the " $\mathrm{K}$ " key when the position of the dot relative to the landmarks changed, and to press the "D" key when the location stayed the same. One second after their response, the next trial began.

\section{Biased Points $\otimes$ Control Points}

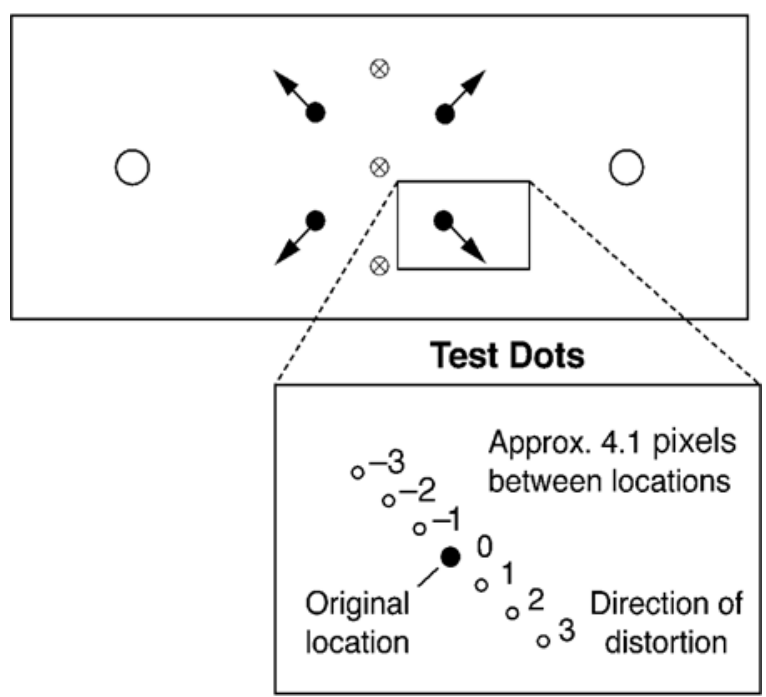

Figure 3. Location of biased and control points relative to the two landmarks in Experiment 2. The arrows show the direction of distortion for the biased points (black dots) as observed in Experiment 1 . For the control points (open dots), no distortion was found. The test dot locations for the lower-right biased point are shown in the box below. 
Participants received a training block of 32 trials to acquaint them with the task. In half of the trials, the position of the dot was not changed, and in the other half of the trials, the dot was moved 12 pixels $\left(.28^{\circ}\right)$ either in the direction of the distortion or in the opposite direction. This served to induce the expectancy that the proportion of no-change trials would be $50 \%$, whereas in the following 10 experimental blocks only every seventh trial was a no-change trial. Due to this manipulation the participants showed approximately equal proportions of change and no-change responses. Each experimental block consisted of 224 trials, in which the four biased points and four control points were counterbalanced with the seven possible changes of the dot location and the four retention intervals. After each experimental block the participants were provided with feedback about their discrimination performance in the form of $d$. Five of these blocks were done in the first, and the other half were done in the second session. Sessions were separated by at least 1 day.

\section{Results}

The data of 1 participant were excluded from further analysis because of poor discrimination $\left(d^{\prime}=0.35\right.$, as opposed to an average of the other participants of $d^{\prime}=1.56$, $S D=0.33$ ). We evaluated the responses separately for the biased and the control points, but collapsed the data over the four positions within each group. For the biased points, the test dots in the direction of the distortions were labeled $+3,+2$, and +1 . Because there were no distortions for the control points in Experiment 1, we chose a conservative assignment: We labeled the test dots on the side with a higher number of no-change responses as +3 , +2 , and +1 . It turned out that for all control points the test dots on the left side received more no-change responses.

As can be seen in Figure 4, the response pattern for the control points is symmetrical. The rate of no-change responses differs only $3 \%-17 \%$ between the two directions for the four retention intervals $[|t(10)| \leq 1.65, p>.10]$. For the biased points, the participants showed $42 \%-63 \%$ more no-change responses in the direction of the distortion, as would be expected if their representation had shifted in this direction $[t(10)>2.66, p<.05$, for all retention intervals].

To investigate distortion, accuracy, and the decision criterion independently, we obtained estimates for the parameters $\mu_{m}, \sigma_{m}$, and $\kappa$ (see Appendix) individually for every participant and retention interval. In most cases, the model provided a good fit for the data. However, in 17 of the 88 fits the residual error differed significantly $(\alpha=$ $5 \%$ ) from zero.

The average estimates for the three parameters (Figure 5) were submitted to a repeated measures ANOVA with the variables retention interval and point type (biased vs. control). The $\mu_{m}$ values for the biased points were significantly different from those of the control points $[F(1,10)=$ $\left.8.77, M S_{\mathrm{e}}=5.49, p=.014\right]$. Already after $100 \mathrm{msec}$ the $\mu_{m}$ estimates for the biased points differed significantly from zero $[t(10)=2.74, p=.021]$, and rose further from the 100 -msec to the 200-msec condition $[t(10)=2.37$, $p=.025]$. We found no other significant increases. For the control points, no significant distortion was found.

The $\sigma_{m}$ parameter showed an effect of retention interval $\left[F(3,30)=5.30, M S_{\mathrm{e}}=0.41, p=.005\right]$. There was, however, no difference between the biased points and the control points in this parameter $\left[F(1,10)=0.36, M S_{\mathrm{e}}=0.74\right.$, $p=.853]$, nor was there an interaction between point type and retention interval $\left[F(3,30)=1.23, M S_{\mathrm{e}}=0.20\right.$, $p=.31]$. The decision criterion $\mathrm{\kappa}$ was standardized with respect to the $\sigma_{m}$ value of the same condition before it was submitted to the ANOVA. The standardized $\kappa$ did not change with retention interval $\left[F(3,30)=0.722, M S_{\mathrm{e}}=0.186\right.$, $p=.54]$, but was significantly stricter for the control points than for the biased points $\left[F(1,10)=6.23, M S_{\mathrm{e}}=0.126\right.$, $p=.032]$. There was also a significant retention interval $\times$ point type interaction for the standardized $\kappa[F(3,30)=$ $\left.3.41, M S_{\mathrm{e}}=0.035, p<.03\right]$.

\section{Discussion}

The results of Experiment 2 clearly indicate that observers misjudged the location of the biased points after only $100 \mathrm{msec}$. For test dots in the direction of the distortion, the number of false no-change responses was higher than in the opposite direction. Accordingly, the size of the distortion $\left(\mu_{m}\right)$ was significantly different from zero. This effect was observable for all retention intervals. A significant distortion was thus present after just a $100-\mathrm{msec}$

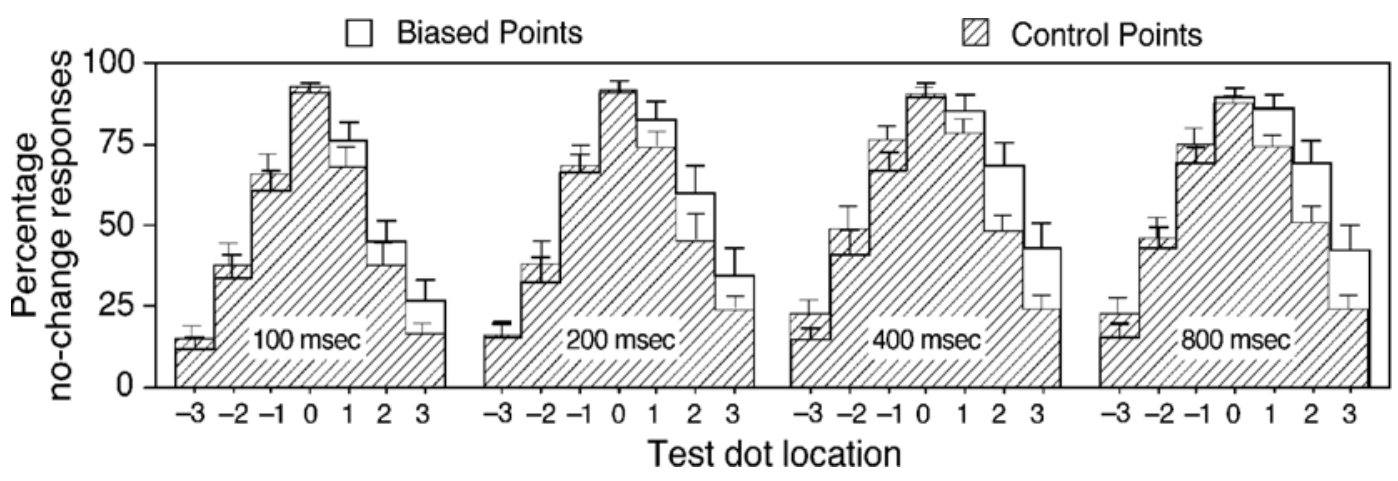

Figure 4. Percentage of no-change responses for biased and control points dependent on test dot location in Experiment 2 . The results for different retention intervals are shown separately. The error bars represent standard errors. 

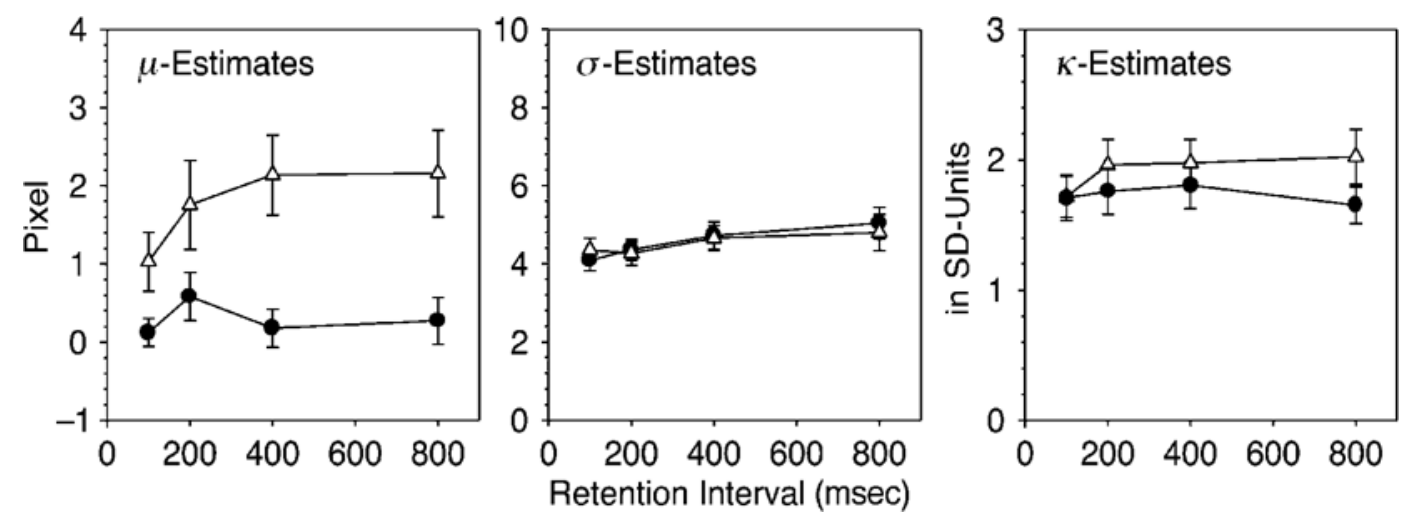

Figure 5. Mean estimates for $\mu_{m}, \sigma_{m}$, and $\kappa$ derived from the results of Experiment 2. The parameter $\mu_{m}$ is a measure for the size of the distortion, $\sigma_{m}$ for accuracy of the memory, and $\kappa$ for the decision criterion of each participant. The parameter $\kappa$ is presented in $S D$ units of the respective $\sigma_{m}$. Parameter estimates were computed individually for each participant. The error bars represent standard errors.

delay between the two stimulus presentations, increasing with longer delays. Unlike the biased points, the three control locations did not show any systematic distortion. The uncertainty of the remembered location $\left(\sigma_{m}\right)$ increased with increasing delay for all locations. This finding indicates that information about the presented location is lost over time whether the memory of the location is distorted or not. However, participants applied a stricter decision criterion when making no-change responses for control points. This might be due to the fact that the control locations were aligned with the midpoint between the landmarks, increasing confidence in the judgments.

In sum, the results of the second experiment suggest that the same spatial memory distortions were identifiable with a visual discrimination task as with the reproduction task in Experiment 1. However, due to the brief stimulus exposures, other processes might also have contributed to the spatial distortions found. For example, O'Regan (1984) found that the position of a brief and peripherally presented stimulus is misjudged to be closer to the fixation point. In our experiment, however, the distortion went outward from the average fixation point, assuming that participants fixated the midpoint between the two landmarks. In a relative localization task, on the other hand, the position of a smaller object relative to a larger one is judged to be more peripheral (Müsseler, van der Heijden, Mahmud, Deubel, \& Ertsey, 1999), but only under successive presentations of the larger and smaller stimuli. Even though these findings do not offer an alternative explanation of our results, we changed the procedure in the third experiment to rule out any potential effects of the different dot locations between biased and control points.

\section{EXPERIMENT 3}

Experiment 3 addressed two concerns that arise with the interpretation of the previous experiment. First, control and biased points had different eccentricities; thus differences in the distortion may have been due to the retinal position rather than to the influence of the landmarks. We addressed this problem by using only the four biased points with and without presentation of the landmarks. If the distortion was due to the presence of the landmarks, it should disappear if the dot is presented alone at the same eccentricity. By using identical test dot locations, this experiment allowed us to rule out any explanations of the distortion effect based solely on eccentricity or eye movements in the direction of the test dot locations, because these should be the same between the two conditions.

Second, in the previous experiment participants were led to expect an equal number of change and no-change trials. During practice, the ratio of change and no-change trials was $1: 1$, with immediate feedback about the correctness of each response after each trial. Participants subsequently reported approximately as many change as no-change responses, although only every seventh trial was a no-change trial. Using this manipulation we were able to gather the optimal amount of information from the data. However, because our results are based on parameter estimations from a formal model, we wanted to ensure that estimates for $\sigma_{m}$ and especially of $\mu_{m}$ are independent of the response criterion $\kappa$. We therefore assigned participants to two different expectancy conditions, in which the response criterion was manipulated by changing the subjects' expectancies regarding the correct proportion of trial types. For half the participants, the practice block now reflected the correct ratio of no-change to change trials of $1: 6$, whereas for the other half the ratio remained 1:1, as in Experiment 2. If our previous results are independent of the criterion, this manipulation should only lead to a change in parameter $\kappa$, leaving the estimates for the other parameters unchanged.

\section{Method}

Participants. Twenty students participated in the experiment for 30 DM reimbursement. Their age ranged from 22 to 35 years. 
Apparatus and Stimuli. The setup was identical to that of Experiment 2 with the exception that only the four biased locations were used in this experiment.

Procedure. The procedure was to a large degree identical to that of Experiment 2. There were two landmark conditions, alternating between blocks: In the landmarks-visible condition, the original dot and the test dot were both presented together with the two landmarks. In the no-landmarks condition, the original and the test dot were both shown without the landmarks. The sequence of conditions was alternated from participant to participant. After each block, the participants received feedback about their discrimination performance. The interval between the first and the second presentations was reduced to 50,100,200, or $400 \mathrm{msec}$ and was varied randomly within a block. To ensure that no apparent motion was perceived even in the shortest retention interval, we used a higher intensity mask. A rectangular region of $300 \times 200$ pixels $\left(7^{\circ} \times 4.6^{\circ}\right)$ was filled with a random checkerboard pattern consisting of individual squares of 4 pixels $\left(5.5^{\prime}\right)$, changing randomly between black and white every $16 \mathrm{msec}$.

Participants were assigned to one of two expectancy conditions, which differed solely in the practice block. In the high-expectancy condition, half of the 32 practice trials were no-change trials, as in the previous experiment, whereas in the low-expectancy condition, the ratio between the change and no-change trials was 1:6. In addition to giving trial-by-trial feedback in the practice block, the experimenter informed the participants in the low-expectancy condition that only one seventh of all trials were no-change trials. Assignment to condition was not randomized because the low-expectancy condition was added to the experiment later.

\section{Results}

The data from 1 participant in the low-expectation condition were excluded from the analysis because of poor performance in the no-landmark condition $\left(d^{\prime}=-0.05\right)$. The other participants in this condition showed a mean performance of $d^{\prime}=0.70, S D=0.51$.

In the landmarks-visible condition, the rate of no-change responses was again significantly higher for changes in the direction of the distortion versus in the opposite direction for all four retention intervals $[t(18)>4.16, p \leq$ .001 ] compared with nonsignificantor significantly lower rates in the no-landmarks condition $[-3.28 \leq t(18) \leq$ $.19, p>.004]$. The model fit the data well. Only 10 of the 152 fits deviated on a $5 \%$-significance level from the data. Because of space limitations we do not include another histogram of the results for this experiment or Experiment 4 as we did for Experiment 2.

The parameter estimates (Figure 6) were submitted to an ANOVA with landmark condition and rentention interval as within-subjects variables and the expectancy condition as a between-subjects variable. The expectancy condition did not have any significant influence on the estimates for $\mu_{m}$, nor was there any significant interaction involving this variable (all $F_{\mathrm{S}}<1$ ). Thus, estimates were robust to big changes in the rate of no-change response, and we present the data collapsed over this factor. The $\mu_{m}$ estimates differed significantly between the landmark conditions $[F(1,17)=$ $\left.70.22, M S_{\mathrm{e}}=4.21, p<.001\right]$ and showed a significant effect of retention interval $\left[F(3,51)=17.41, M S_{\mathrm{e}}=0.68\right.$, $p<.001]$. The landmark condition $\times$ retention interval interaction was significant $\left[F(3,51)=2.89, M S_{\mathrm{e}}=1.03\right.$, $p=.044]$. After a retention interval of only $50 \mathrm{msec}$ the estimates for the distortion in the landmarks-visible condition already differed significantly from zero $[t(18)=$ $3.32, p=.004]$. In the no-landmark condition, the 50-msec and $100-\mathrm{msec}$ condition showed a $\mu_{m}$ value smaller than zero $[t(18)=-2.79, p=.011$, and $t(18)=-2.75, p=$ $.013]$; for the remaining two retention intervals, this difference was not significant. Planned paired comparisons (one-sided) in the landmarks-visible condition revealed no significant increase in distortion from 50 to $100 \mathrm{msec}$ $[t(18)=0.49]$, but significant increases from 100 to $200 \mathrm{msec}[t(18)=3.01, p=.003]$ and 200 to $400 \mathrm{msec}$ $[t(18)=3.55, p=.001]$.

Estimates for $\sigma_{m}$ also changed with increasing retention interval $\left[F(3,51)=15.66, M S_{\mathrm{e}}=0.67, p<.001\right]$. There was a significant difference between the two landmark conditions $\left[F(1,17)=5.34, M S_{\mathrm{e}}=1.53, p=.034\right]$, but no interaction with the retention interval $[F(3,51)=0.57$, $\left.M S_{\mathrm{e}}=0.53, p=.93\right]$. The uncertainty parameter differed significantly between the low- and high-expectancy conditions $\left[F(1,17)=7.67, M S_{\mathrm{e}}=14.77, p=.013\right]$, but no interaction involving this variable was significant.

As expected, the $\kappa$ estimates varied significantly with the expectancy condition $\left[F(1,17)=53.36, M S_{\mathrm{e}}=0.95\right.$, $p<.001]$. In the low-expectancy condition, the estimates
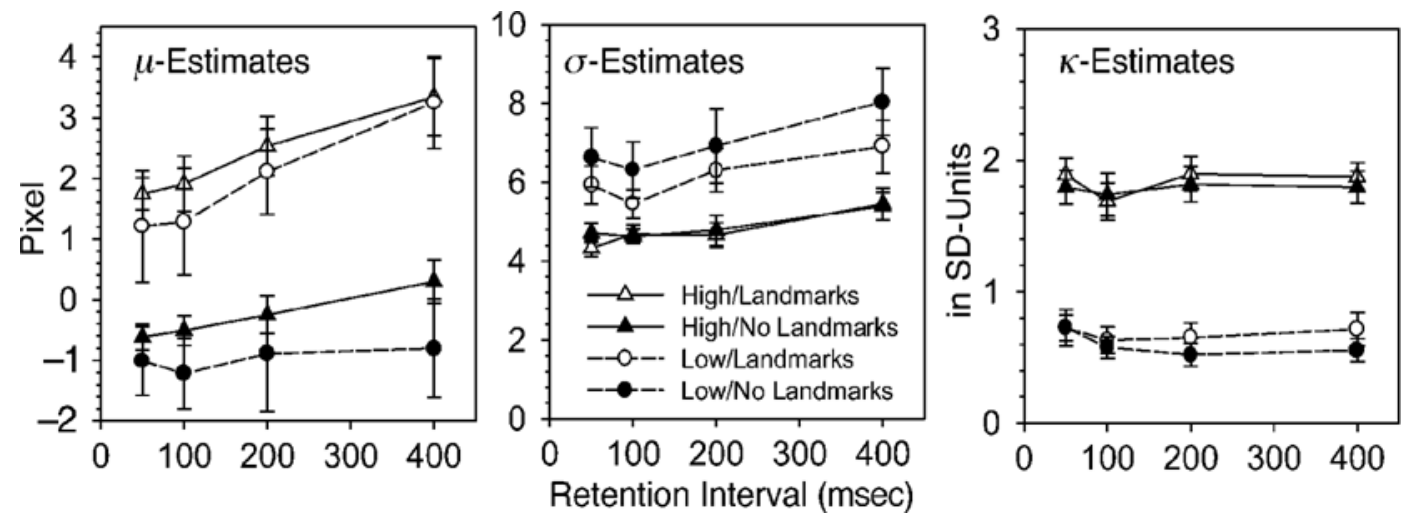

Figure 6. Mean estimates $( \pm S E)$ for $\mu_{m}, \sigma_{m}$, and standardized $\kappa$ in Experiment 3. 
were considerably lower than in the high-expectancy condition, reflecting a stricter criterion for a no-change response. Whereas in the high-expectancy condition $58.5 \%$ of the responses indicated no change, this number decreased to $26.3 \%$ in the low-expectancy condition. There was a significant change of $\kappa$ with retention interval $\left[F(3,51)=2.94, M S_{\mathrm{e}}=0.036, p=.041\right]$, but no significant effect of condition $\left[F(1,17)=2.22, M S_{\mathrm{e}}=0.076\right.$, $p=.15]$. No other interaction was significant.

\section{Discussion}

As in Experiment 2, the results show the presence of a spatial memory distortion following a brief retention interval. Even for retention intervals as brief as $50 \mathrm{msec}$, a significant distortion was observed in the landmarks-visible condition. As expected, the no-landmarks condition did not show a systematic distortion in the same direction as observed with landmarks. This makes the eccentricity of the test locations an unlikely candidate to explain the different spatial distortions. The results thereby also confirm that the spatial organizationinduced by the landmarks is responsible for the distortion. The stronger distortion effect compared with that in Experiment 2 can probably be attributed to the higher intensity mask used in this experiment.

The effect of the retention interval was more pronounced in this experiment than in Experiment 2. With increasing delay, the size of the distortion doubled in the landmarksvisible condition. In terms of the Huttenlocheret al. (1991) model, this could be interpreted as the fast decay of accurate location information within the first $400 \mathrm{msec}$ and a stronger weighting of categorical information in reconstructing the remembered location. The interaction between the landmark-condition and retention interval taken together with Figure 6 indicates that the distortion increased with increasing retention interval, even though the interaction is weak. This is due to the $\mu_{m}$ estimates in both conditions showing a similar pattern with increasing retention interval. In the no-landmarks condition, though, the absolute size of the distortions is very small.

The change in the expectancy of the participants had the predicted result. The number of no-change responses in the low-expectancy condition was only half of that in the highexpectancy condition, which is reflected in lower estimates for the response criterion. There were no differences in estimated distortion between these two conditions, but the uncertainty parameter was affected. This indicates that $\sigma_{m}$ might not be entirely independent of the response criterion.

\section{EXPERIMENT 4}

In Experiments 2 and 3, we showed for a subset of locations that the distortion found in a visual discrimination paradigm was in the same direction as in the reproduction paradigm of Experiment 1. Four locations showed a distortion in an outward direction and three positions showed no distortion in Experiment 1. Although retinal eccentricity is an unlikely explanation for this pattern, given the re- sults of Experiment 3, we tried to replicate in Experiment 4 the distortion pattern of Experiment 1 for distortions toward the center of the display. A finding that our visual discrimination procedure produced the same results as those from Experiment 1 for three different types of distortions (no distortion, outward expansion, compression toward the center) would strengthen our claim that the distortions identified in both tasks are based on the same principles. We therefore chose four new locations that had shown a distortion toward the center in Experiment 1 and tested them in the same way as before.

\section{Method}

Participants. Eleven student participants from the same pool as in the previous experiments took part in this 2-h experiment.

Stimuli and Procedure. The stimulus display and the mask were the same as in Experiment 3. The only change to the previous experiment was the location of the dots. We chose four points $\{ \pm 90$, $\pm 30\}$ close to the landmarks that had shown a distortion toward the center in Experiment 1 (Figure 1). The test dots for these locations were aligned along the mean distortion vector found in Experiment 1 , three in both directions with an average distance of 4.1 pixels between them. We labeled the test dots in the direction of the distortion $+1,+2$, and +3 , and the test dots against the direction of the distortion $-1,-2$, and -3 . As before, a distortion in the direction of Experiment 1 would yield positive $\mu_{m}$ values.

\section{Results}

The data from 1 participant were excluded from the analysis because his performance in the no-landmark condition was equivalent to guessing $\left(d^{\prime}=-0.08\right)$. The performance of the other participants in the no-landmark condition was also poorer than in the last experiment $\left(d^{\prime}=0.68, S D=0.42\right)$, reflecting a more difficult discrimination due to the higher eccentricity of the points. The parameter estimation was performed as in the last experiment. In 11 of 88 cases, the model prediction differed significantly $(\alpha=.05)$ from the data, with 8 of those cases in the landmark-visible condition. The model fit was unstable for 1 participant in the most difficult condition (no landmark, 400-msec retention interval), with guessinglevel performance. We replaced this cell for further analysis with the mean of the other participants in this condition. Exclusion of this participants' data would not have changed the results qualitatively.

As in Experiments 2 and 3, no-change responses again showed significantly higher rates for changes in the direction of the distortion versus in the opposite direction for all four retention intervals in the landmarks-visible condition $[t(8)>2.36, p \leq .046]$. In contrast, the results were symmetric for all retention intervals in the no-landmark condition $\left[t(8) \leq 0.96, p>\right.$.364]. As before, the $\mu_{m}$ estimates (Figure 7) for the landmark differed significantly from those for the no-landmark condition $[F(1,9)=7.47$, $\left.M S_{\mathrm{e}}=4.69, p=.023\right]$ and were also significantly different from zero $[t(9)=2.33, p=.044]$. There was no significant effect of retentioninterval $\left[F(3,27)=2.10, M S_{\mathrm{e}}=0.63\right.$, $p=.12]$, nor was there a retention interval $\times$ condition interaction $\left[F(3,27)=0.77, M S_{\mathrm{e}}=0.48, p=.52\right]$. 

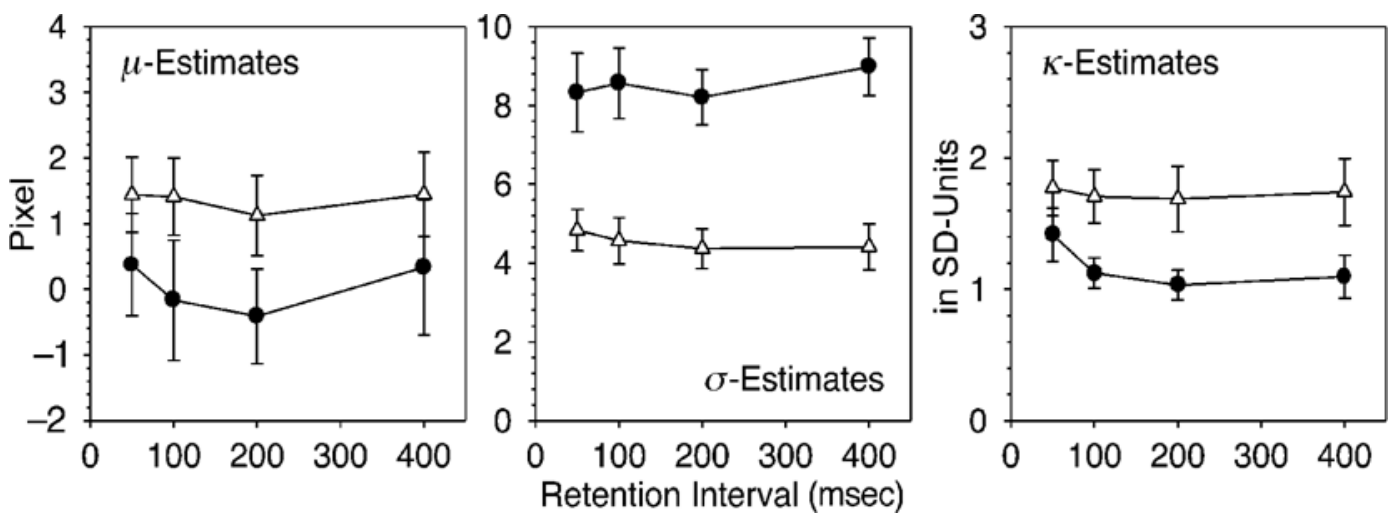

Figure 7. Mean estimates $( \pm S E)$ for $\mu_{m}, \sigma_{m}$, and standardized $\kappa$ in Experiment 4.

The $\sigma_{m}$ estimates showed a large difference between the landmark and the no-landmark conditions $[F(1,8)=45.86$, $\left.M S_{\mathrm{e}}=6.90, p<.001\right]$. There was no effect of retention interval $\left[F(3,27)=0.66, M S_{\mathrm{e}}=0.89, p=.58\right]$, nor an interaction between condition and retention interval $[F(3,24)=$ $\left.0.94, M S_{\mathrm{e}}=1.06, p=.43\right]$. The $\mathrm{\kappa}$-estimates differed significantly between conditions $\left[F(1,9)=23.58, M S_{\mathrm{e}}=\right.$ $0.266, p<.001]$ and varied slightly with retention inter$\operatorname{val}\left[F(3,27)=3.06, M S_{\mathrm{e}}=0.067, p=.045\right]$.

\section{Discussion}

As in all prior experiments, the participants showed evidence of spatial distortions at very brief retention intervals. In this case, positive values corresponded to distortions toward the center, indicating a tendency to remember the dot further toward the middle of the display when landmarks were present. In the no-landmarks condition, no evidence of any distortion was found. The distortion was smaller than in Experiment 2 and 3, just as the original distortion for the more eccentric locations was smaller in Experiment 1.

In contrast to the previous experiments, Experiment 4 did not indicate any change of the distortion for longer retention intervals, and only 1 participant showed any indication of an increasing distortion. In this experiment, the distortion thus seemed to be fully present at $50 \mathrm{msec}$, and the data thus do not suggest an increase of distortion with increasing retention interval, as in the previous experiment.

The $\sigma_{m}$ estimates were substantially higher in the nolandmark condition than in the landmarks-visible condition and were also much higher than for the more central points previously used. This increased uncertainty in the underlying memory representations probably reflects the higher eccentricity of the points. The much lower $\sigma_{m}$ estimates in the landmark condition can be attributed to the proximity of the landmark to the dots, helping the participants to anchor their spatial representation. A similar argument can be made for the decreased criterion used in the landmarks condition. We assume that this difference might reflect an adjustment toward a stricter criterion when the task becomes easier.

\section{GENERAL DISCUSSION}

The series of experiments presented in this article addresses two important questions regarding the time course of spatial memory distortions - the point at which spatial distortions are first detectable and their development over time. As a prerequisite, we need to show that the visual discrimination task used in Experiments 2, 3, and 4 taps into the same cognitive processes as those tapped by the traditionally employed reproduction method used in Experiment 1 . The close resemblance of the pattern of results across the two different types of tasks suggests this to be the case. Dot locations, which were reproduced away from the center of the display in Experiment 1, also showed an outward distortion in our discrimination paradigm, whereas control points that showed no distortion in the reproduction task similarly showed no distortion in the discrimination paradigm. Dot locations near the landmarks, which showed a distortion toward the center of the display in the reproduction task, exhibited the same distortion in the discrimination task. As expected, the presence of the landmarks was crucial for these distortions to occur-no distortions or small distortions in the opposite direction were found in conditions where no landmarks were presented. Taken together, the high agreement of these two measures across different locations and conditions lets us propose that the visual discrimination method used in this study can be validly taken as a measure for spatial memory distortions.

The main finding of our study consists of the fast onset of spatial distortions. In Experiments 3 and 4, distortions were detectable at a 50-msec retention interval between the first presentation and the second presentation of the stimulus. The distortion pattern of reproduced dot locations in Experiment 1 can therefore be linked to distortions, which are present almost immediately after a 50-msec retention interval. 
In our view, the distortions reported in all four experiments are memory based and not solely due to misperceptions of spatial relations. First, unlike in many other studies dealing with perceptual biases, the visual display when the stimulus location was encoded was identical to the visual display when the location had to be reproduced (Experiment 1) or when a judgment about the location had to be made (Experiments 2-4). If we assume that a dot near a landmark is perceived as further away from the landmark than it really is, this would, of course, lead to a biased memory representation of the location of the dot. However, when participants reproducing the dot location or judging the relative location of the dot in the same visual scene, the same perceptual processes are at work as during the original encoding of the location. Any systematic effects of perceptual biases therefore should cancel out between encoding and reproduction as long as the perceptual context and the perceptual processes stay the same. Only when the perceptual situations during encoding and reproduction differ significantly should perceptual biases play a role in reproduction experiments (see Crawford et al., 2000, for a similar point). In the tilt illusion, for example, this is achieved by presenting a line together with an illusion-inducing context (usually a second, disoriented line) while the test line's orientation has to be reproduced without the presence of the context (Wenderoth \& Johnson, 1985).

Assuming a perceptual bias as the source of our results thus implies that the visual situation between first and second presentations must change, either by a change in fixation or by a change in the allocation of attention. A recent study by Suzuki and Cavanagh (1997) provides evidence that the allocation of attention can influence position judgments. In their study, observers had to judge the relative position of two vertical lines (vernier stimulus) after attending to briefly presented cues to the sides of the lines. The two lines were presented simultaneously, and thus their task did not include a memory component. Their results show that the perceived relative position of the two lines is biased away from an attended cue presented prior to the vernier stimulus. In addition, Suzuki and Cavanagh were able to show that this repulsion effect away from an attended location developed within the first $200 \mathrm{msec}$ after the presentation of the cue and was already present within $50 \mathrm{msec}$. Unlike our data, however, their results also showed that the effect of attention slowly diminishes over the course of the first $800 \mathrm{msec}$. The different time courses therefore make it implausible that attentional factors can account for our results. Recent results by Sheth and Shimojo (2001) also make eye movements unlikely candidates for the spatial distortions we found in our experiments.

A second argument in favor of a memory interpretation of the results concerns the time course found in Experiments 2 and 3, where an increase of the retention interval led to an increase of distortion. In Experiment 3, moreover, the increase of distortion in the landmarks-visible condition was significantly different from the slight distortions in the no-landmarks condition. The dependence of an effect on retention time has traditionally been interpreted as an indication for a role of memory in the effect. Even though the results from Experiment 4 fail to support a general increase of spatial distortion with increasing retention interval, independent reports of increasing visual distortions with increasing retention interval support a memory effect interpretation (Crawford et al., 2000; Sheth \& Shimojo, 2001).

However, the lack of an increase of the spatial distortion in Experiment 4, together with the weak interaction found between retention interval and landmark condition in Experiment 3 , allows for an alternative explanation of our results. ${ }^{1}$ The change of the distortion with increasing retention interval and the bias induced by the landmarks may depend on two independent processes. An immediate (within $50 \mathrm{msec}$ ) development of a spatial bias congruent with the results of Experiment 1 would be driven by the presence of landmarks. A slower process, which is independent of the landmarks, would cause the drift away from the center of the display, causing the increases in absolute distortion that we found in Experiments 2 and 3. The similar drift of the remembered location for the landmarkvisible and no-landmark conditions in Experiment 3 lends some credibility to this point. Even though there is no noticeable absolute distortion in the no-landmarks condition, the graphs run almost parallel. Therefore, if one conceives of the distortion as the relative difference between the two conditions, there seems to be little change dependent on retention interval (in our previous discussions, we have mainly focused on the absolute error as an indication of distortion). At this point we cannot exclude this alternative explanation. The origin of this hypothetical process, however, remains unclear. It seems unlikely that a drift of the memory representation in an outward direction is connected to the eccentricity of the presented location. In Experiment 4 , in which the target dots were at even more peripheral positions than in Experiments 2 and 3, no change in the distortion was found.

In any case, our results clearly emphasize the close link between perceptual and memory processes in the temporal domain, showing that spatial memory is distorted after a retention interval of $50 \mathrm{msec}$. Within the framework of Huttenlocher et al.'s (1991) model of reconstructive spatial memory, this indicates that categorical information immediately plays a role in remembering spatial locations once the perceptual input is gone. A part of the coordinate information, in contrast, must deteriorate very rapidly after stimulus offset.

According to the idea of two contributing representations, the time course of the distortion is determined by a shift in the relative weights between the two sources of information. A representation of the stimulus in terms of the perceived structure of space (e.g., its category) is encoded very rapidly within a few hundred milliseconds after the onset of a stimulus, increasing the weight of this informa- 
tion. Simultaneously, detailed visuospatial information in iconic memory quickly fades (see Coltheart, 1980, for a review), decreasing the weight of this source of information. The presence of an almost immediate distortion fits well with the concept of a rapid loss of information from the visual buffer once the visual stimulus is masked (e.g., Gegenfurtner \& Sperling, 1993). When comparing the two perceptual events, observers thus have to reconstruct the first event from a short-term memory representation, whereas the second perceptual event can rely more heavily (maybe exclusively) on the available visual input as a source of location information.

Studying the time course of distortions in spatial representations over very short retention intervals raises the question whether participants may have been able to use additional sources of information at very short retention intervals. One possible candidate for such information is apparent motion. Our participants could have followed the simple strategy of reporting a change whenever they perceived a motion signal between the first and the second presentations of the dot. This could have caused the reduced spatial distortion for brief retention intervals, even though the positional information was already distorted. As results by Gros, Pope, and Cohn (1996) show, a motion signal can be extracted and used for the judgment about relative position for an interstimulus interval (ISI) of up to $100 \mathrm{msec}$. However, in their experiment the ISI was unmasked. Using a static visual noise mask, Braddick (1973) was able to reduce the maximum ISI at which apparent motion was still visible to approximately $20 \mathrm{msec}$, well below the 50-msec retention interval we used. Our dynamic visual noise mask, which provides multiple random motion signals across the whole stimulus display, should have led to an even lower maximum ISI for apparent motion.

In sum, our results clearly show that distortions in the spatial representation are present after retention intervals of only $50 \mathrm{msec}$. Although it is difficult to locate the precise point in visual processing at which the distortions occur, the short lag in processing between the two perceptual events suggests that they are caused by memory effects early in the stream of perceptual processing. Our results indicate clearly that an accurate representation of the location of the dot is lost very early. All information entering shortterm memory seems to be already subject to a distortion. Although less clear, our results also indicate that the size of the distortion is then increasing further. Whether additional perceptual biases are also present in early visual processing of our stimulus displays is an open question.

The present results raise the interesting question of whether spatial memory distortions usually attributed to long-term memory can be accounted for by early processes. The pattern of distortions found in Experiment 1 is the same as the pattern of distortions found for children searching for a buried toy in a rectangular sandbox (Huttenlocher et al., 1994). It thus seems that the spatial distortions developing within the first $50 \mathrm{msec}$ after the presentation of a visual stimulus do not change qualitatively over the time span of many seconds or even minutes. The distortions produced by the hierarchical structure of spa- tial memories for large-scale spaces, acquired over long periods of time (Hirtle \& Jonides, 1985), closely resemble the effects of structuring visually perceived space in our experiments. This implies that similar principles might be at work in mental representations of space, at both long and very brief time scales.

\section{REFERENCES}

BRADDICK, O. (1973). The masking of apparent motion in random-dot patterns. Vision Research, 13, 355-369.

Bryant, D. J., \& Subbiah, I. (1993). Strategic and perceptual factors producing tilt contrast in dot localization. Memory \& Cognition, 21, 773-784.

Carmichael, L., Hogan, H. P, \& Walter, A. A. (1932). An experimental study of the effect of language on the reproduction of visually perceived forms. Journal of Experimental Psychology, 15, 73-86.

Coltheart, M. (1980). Iconic memory and visible persistence. Perception \& Psychophysics, 27, 183-228.

Crawford, L. E., Huttenlocher, J., \& Engebretson, P. H. (2000). Category effects on estimates of stimuli: Perception or reconstruction? Psychological Science, 11, 280-284.

Crumbaugh, J. C. (1954). Temporal changes in the memory of visually perceived form. American Journal of Psychology, 67, 647-658.

DIEDRICHSEN, J. (1999). Gibt es ein motorisches Kurzzeitgedächtnis für Positionen im Greifraum? Unpublished diploma thesis, University of Göttingen, Germany.

Freyd, J. J., \& Johnson, J. Q. (1987). Probing the time course of representational momentum. Journal of Experimental Psychology: Learning, Memory, \& Cognition, 13, 259-268.

Gegenfurtner, K.R., \& Sperling, G. (1993). Information transfer in iconic memory experiments. Journal of Experimental Psychology: Human Perception \& Performance, 19, 845-866.

Gros, B. L., Pope, D. R. \& CoHn, T. E. (1996). Relative efficiency for the detection of apparent motion. Vision Research, 36, 2297-2302.

Hirtle, S. C., \& Jonides, J. (1985). Evidence of hierarchies in cognitive maps. Memory \& Cognition, 13, 208-217.

Huttenlocher, J., Hedges, L. V., \& Duncan, S. (1991). Categories and particulars: Prototype effects in estimating spatial location. Psychological Review, 98, 352-376.

Huttenlocher, J., Newcombe, N., \& Sandberg, E. H. (1994). The coding of spatial location in young children. Cognitive Psychology, 27, 115-147.

Laeng, B., Peters, M., \& McCabe, B. (1998). Memory for locations within regions: Spatial biases and visual hemifield differences. Memory \& Cognition, 26, 97-107.

LEVInson, S. (1996). Frames of reference and Molyneux's question: Crosslinguistic evidence. In P. Bloom, M. A. Peterson, L. Nadel, \& M. F. Garrett (Eds.), Language and space (pp. 109-107). Cambridge, MA: MIT Press.

Moore, C. M., \& Egeth, H. (1997). Perception without attention: Evidence of grouping under conditions of inattention. Journal of Experimental Psychology: Human Perception \& Performance, 23, 339-352.

Müsseler, J., van der Heijden, A. H. C., Mahmud, S. H., Deubel, H., \& ERTSEY,S. (1999). Relative mislocalization of briefly presented stimuli in the retinal periphery. Perception \& Psychophysics, 61, 1646-1661.

Nelson, T. O., \& Chaiklin, S. (1980). Immediate memory for spatial location. Journal of Experimental Psychology: Human Learning \& Memory, 6, 529-545.

O'Regan, J. K. (1984). Retinal versus extraretinal influences in flash localization during saccadic eye movements in the presence of a visible background. Perception \& Psychophysics, 36, 1-14.

Riley, D. A. (1962). Memory for form. In L. Postman (Ed.), Psychology in the making (pp. 402-465). New York: Knopf.

Schiano, D. J., \& TVERSKY, B. (1992). Structure and strategy in encoding simplified graphs. Memory \& Cognition, 20, 12-20.

SCHMidT, T., Werner, S., \& DiedrichSEN, J. (2002). Spatial distortions induced by multiple visual landmarks: Evidence for a partition principle. Manuscript submitted for publication.

Sheth, B.R. \& Shimojo, S. (2001). Compression of space in visual memory. Vision Research, 41, 329-341. 
SoKal, R. R., \& RoHLF, F. J. (1981). Biometry: The principles and practice of statistics in biological research (2nd ed.). San Francisco: W. H. Freeman.

Stevens, A., \& Coupe, P. (1978). Distortions in judged spatial relations. Cognitive Psychology, 10, 422-437.

Suzuki, S., \& Cavanagh, P. (1997). Focused attention distorts visual space: An attentional repulsion effect. Journal of Experimental Psychology: Human Perception \& Performance, 23, 443-463.

Tversky, B. (1981). Distortions in memory for maps. Cognitive Psychology, 13, 407-433.

Wenderoth, P., \& Johnson, M. (1985). What is the appropriate control for the tilt illusion? Perception, 14, 275-283.
Werner, S., \& Schmidt, T. (2000). Investigating spatial reference systems through distortions in visual memory. In C. Freksa, W. Brauer, C. Habel, \& K. F. Wender (Eds.), Spatial cognition II-Integrating abstract theories, empirical studies, formal methods, and practical applications (LNAI 1849, pp. 169-183). Berlin: Springer.

WulfF, F. (1922). Beiträge zur Psychologie der Gestalt: Über die Veränderung von Vorstellungen. Psychologische Forschung, 1, 333373.

\section{NOTE}

1. We thank an anonymous reviewer for this suggestion.

\section{APPENDIX \\ Model for the Estimation of Memory Distortions From a Visual Discrimination Paradigm}

We assume that the remembered location is a normally distributed random variable $m$ with mean $\mu_{m}$ and standard deviation $\sigma_{m}$. The test dot is presented at the position $T_{i}$, and we posit that it is perceived without error. If the remembered location $m$ falls within the boundaries $T_{i}-\kappa$ and $T_{i}+\kappa$, with $\mathrm{\kappa}$ being the criterion for the decision process, the participant will report no change in the display. The probability for a no-change response given a comparison stimulus $T_{i}$ thus equals

$$
P\left(\text { nochange } \mid T_{i}\right)=\int_{T_{i-\kappa}}^{T_{i+\kappa}} \frac{1}{\sqrt{2 \pi \sigma_{m}^{2}}} e^{-\frac{\left(x-\mu_{m}\right)^{2}}{2 \sigma_{m}^{2}}} d x .
$$

We estimated the parameters $\mu_{m}, \sigma_{m}$, and $\kappa$ for each participant and condition by predicting the frequency of change and no-change responses for each test dot. The deviation from the observed frequencies was measured by the error term $G$, which is defined as the log-likelihood ratio of the predicted and observed frequencies (see Sokal \& Rohlf, 1981). An iterative fitting procedure was employed to minimize $G$ by adjusting the three free parameters.

Note-We thank Walter Zucchini, Department for Statistics, University of Göttingen, for helpful suggestions in the development of this model.

(Manuscript received August 3, 1999; revision accepted for publication April 3, 2002.) 